INPLASY

PROTOCOL

To cite: Jafrin et al. Insight into the Role of IL-1 1 rs 1143634 (+3954C>T) Polymorphism in Cancer Risk: An Updated Meta-analysis and Trial Sequential Analysis. Inplasy protocol 2021100044. doi: 10.37766/inplasy2021.10.0044

Received: 14 October 2021

Published: 15 October 2021

Corresponding author: Mohammad Safiqul Islam

research_safiq@yahoo.com

Author Affiliation:

Noakhali Science and

Technology University.

Support: No funding.

Review Stage at time of this submission: Data analysis Completed but not published.

Conflicts of interest:

None declared.

\section{Insight into the Role of IL-1 $\beta$ rs1143634 (+3954C>T) Polymorphism in Cancer Risk: An Updated Meta- analysis and Trial Sequential Analysis}

Jafrin, S1; Aziz, MA2; Islam, MS³.

Review question / Objective: To assess the link of IL-1 $\beta$ rs1143634 (+3954C>T) Polymorphism with cancer.

Condition being studied: The included studies must contain 1) genotypic information and detailed data of IL-1 $\beta$ rs1143634 (+3954C>T) polymorphism 2) case-control studies.

Information sources: PubMed, Google Scholar, CNKI, Web of Science, and EMBASE.

INPLASY registration number: This protocol was registered with the International Platform of Registered Systematic Review and Meta-Analysis Protocols (INPLASY) on 14 October 2021 and was last updated on 14 October 2021 (registration number INPLASY2021100044).

\section{INTRODUCTION}

Review question / Objective: To assess the link of IL-1 $\beta$ rs1143634 (+3954C>T) Polymorphism with cancer.

Rationale: Many individual case-control studies were conducted on IL-1 $\beta$ rs1143634 polymorphism and cancer susceptibility in different ethnicities over decades. Although some studies reported a significant link between this variant and different cancers, others failed to establish any significant association. In this meta-analysis, we summarized the previous studies on the connection of IL-1 $\beta$ rs1143634 polymorphism with cancers to investigate the link of the mentioned SNP on different 
cancers and provide a comprehensive outcome.

Condition being studied: The included studies must contain 1) genotypic information and detailed data of IL-1 $\beta$ rs1143634 (+3954C>T) polymorphism 2) case-control studies.

\section{METHODS}

Search strategy: Multiple authorized electronic databases (PubMed, Google Scholar, CNKI, Web of Science, and EMBASE) were comprehensively searched for related literature extraction processes using specific key terms up to May 2021. The selected key terms included: 'cancer', 'interleukin-1 beta', 'rs1143634 (+3954C>T)', 'IL-1 $\beta$ polymorphism and cancer', 'link between IL-1 $\beta$ rs1143634 and carcinogenesis', 'IL-1 $\beta$ polymorphism and cancer development in various ethnic population'.

Participant or population: Global population.

Intervention: Association of polymorphism was detected as Odds ratio with $95 \%$ confidence interval.

Comparator: Different genotypes and allele frequency of cases were compared with controls.

Study designs to be included: Case control studies with the respective genotyping data of the mentioned SNP.

Eligibility criteria: Case control studies with the respective genotyping data of the mentioned SNP.

Information sources: PubMed, Google Scholar, CNKI, Web of Science, and EMBASE.

Main outcome(s): Our meta-analysis revealed that the presence of IL-1 $\beta$ rs1143634 polymorphism increases the risk of cancer development. Among the polymorphism carriers, the Asian population holds more risk than the other ethnic population.

Quality assessment / Risk of bias analysis: Data analysis was performed in seven genetic models. Sensitivity analysis and publication bias were calculated.

Strategy of data synthesis: Data collected from published articles and meta-analyses were performed with RevMan.

Subgroup analysis: Ethnicity, cancer types, and control source-based subgroup were performed.

Sensitivity analysis: Sensitivity analysis was performed by eliminating each study one by one and found no significant change in the pooled odds ratio.

Language: English.

Country(ies) involved: Bangladesh.

Keywords: Interleukin; IL-1ß; Cancer; Polymorphism; Meta-analysis.

Contributions of each author:

Author 1 - Sarah Jafrin.

Email: sarahjafrin1215@gmail.com

Author 2 - Md. Abdul Aziz.

Email: aziz.nstupharma@gmail.com

Author 3 - Mohammad Safiqul Islam.

Email: research_safiq@yahoo.com 